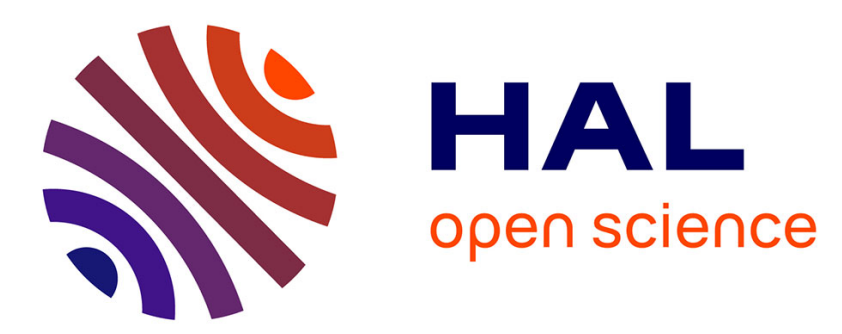

\title{
Robust adaptive control of switched-reluctance motors without velocity measurements
}

Erik Chumacero, Antonio Loria, G. Espinosa-Pérez

\section{To cite this version:}

Erik Chumacero, Antonio Loria, G. Espinosa-Pérez. Robust adaptive control of switched-reluctance motors without velocity measurements. 12th European Control Conference (ECC 2013), Jul 2013, Zurich, Switzerland. pp.4586-4591, 10.23919/ecc.2013.6669158 . hal-00831377

\section{HAL Id: hal-00831377 https://hal.science/hal-00831377}

Submitted on 6 Jun 2013

HAL is a multi-disciplinary open access archive for the deposit and dissemination of scientific research documents, whether they are published or not. The documents may come from teaching and research institutions in France or abroad, or from public or private research centers.
L'archive ouverte pluridisciplinaire HAL, est destinée au dépôt et à la diffusion de documents scientifiques de niveau recherche, publiés ou non, émanant des établissements d'enseignement et de recherche français ou étrangers, des laboratoires publics ou privés. 


\title{
Robust adaptive control of switched-reluctance motors without velocity measurements
}

\author{
Erik Chumacero Antonio Loría Gerardo Espinosa-Pérez
}

\begin{abstract}
We present a speed-sensorless tracking controller for switched reluctance motors with unknown parameters. Our approach relies on the design of two control loops: an outer control-loop for the rotor dynamics which is driven by a PIDtype controller where the stator currents are viewed as virtual control inputs, and an inner tracking control-loop for the stator currents. We assume that the parameters of the rotor (inertia and the load torque) are unknown and we establish uniform global exponential stability. In the case that also the stator parameters are unknown, we add an adaptation law and we establish convergence of the tracking errors.
\end{abstract}

\section{INTRODUCTION}

Switched Reluctance Machines (SRM) are attractive since they are reliable, relatively cheap and they produce high torque at low speed, which makes them suitable for directdrive applications. However, even under experimentallyvalidated assumptions leading to some simplifications, the dynamic model is highly nonlinear $e . g$., the generated electromagnetical torque is a quadratic function of the electric currents and rotor positions. In addition, reliable and accurate indirect sensing methods for the mechanical variables are fundamental in the development of low-cost, highperformance SRM drives; on one hand the use of mechanical sensors increase the cost of the set-up and on the other, velocity sensors are often contaminated with noise. Therefore, avoiding the use of angular velocity and position sensors which is well known as sensorless control, is beyond pure theoretical interest.

There exist a large number of efficient heuristically-based and experimentally-validated control approaches to reduce the number of mechanical sensors in the loop -see e.g., [1], [2], [3], [4], [5]. However, articles on control of switchedreluctance drives that include a rigorous stability analysis, especially in a sensorless context, are rare. The main result in [6] establishes global asymptotic stability for a passivitybased controller in the case of unknown load however, it uses both $^{1}$ mechanical variables. A proportional-derivative-based controller is proposed in [7] but relying on the knowledge of the torque load. In [8] the authors consider only the rotor dynamics that is, it is assumed that the currents are valid physical control inputs. An adaptive position-feedback controller is presented. The controller uses a dirty derivatives filter instead of velocity measurements and it is guaranteed

\footnotetext{
A. Loría is with CNRS. E. Chumacero is with Univ. Paris Sud. Address: LSS-SUPELEC, 91192 Gif-sur-Yvette, France. E-mail: antonio.lorialss.supelec.fr, erik. chumacero@lss.supelec.fr

G. Espinosa-Pérez is with DEPFI - UNAM, A.P. 70-256, 04510 México D.F., MEXICO. gerardoelservidor.unam.mx.

${ }^{1}$ Angular velocity and position measurements
}

that the tracking errors converge to zero. An interesting experimental validation is presented as well to compare the performance against a full-state feedback controller.

In this paper we establish uniform global exponential stability for the SRM under position-feedback control. We use the full model, including both the rotor and stator dynamics and we assume that the rotor inertia and the load torque are unknown. Then, under the assumptions that the rest of the physical parameters are unknown we establish uniform global stability and the convergence of the tracking errors to zero. Our control approach relies on a minimal parameterization, a tracking controller for the stator dynamics and the so-called $\mathrm{PI}^{2} \mathrm{D}$ controller, introduced in [9], for the rotor dynamics. This controller is of a PID type except that the velocity measurements are replaced by dirty derivatives.

The rest of the paper is organized as follows. In next section we briefly describe the motor model, in Section III we discuss the rationale behind our main results, presented in Section IV. In Section V we show some simulation results and we conclude with a few remarks in Section VI.

\section{THE MOTOR MODEL}

After experimental evidence, it is well accepted that the three stator phases of a switched-reluctance motor may be assumed to be magnetically decoupled i.e., the mutual inductance among stator phases is negligible [10]. Furthermore, based on the assumption that the machine operates at relatively low current levels, it is common practice to express the inductance of each phase as a strictly positive Fourier series truncated at the first harmonic that is,

$$
L_{j}(q)=\ell_{0}-\ell_{1} c_{j}, \quad c_{j}:=\cos \left(N_{r} q-(j-1) \frac{2 \pi}{m}\right)
$$

where $q$ denotes the rotor angular position, $\ell_{0}>\ell_{1}>0$ are inductance values and $N_{r}$ is the number of poles. Under these assumptions the dynamic model for the stator currents is given by

$$
u_{j}=L_{j}(q) \dot{x}_{j}+K_{j}(q) \omega x_{j}+R x_{j}
$$

where the index $j \in\{1, \ldots, m\}$ with $m$ is the number of phases, $x_{j}$ denotes currents,

$$
K_{j}(q)=\frac{\partial L_{j}}{\partial q}=N_{r} \ell_{1} s_{j}, \quad s_{j}:=\sin \left(N_{r} q-(j-1) \frac{2 \pi}{m}\right)
$$

corresponds to the phase-inductance variation, $u_{j}$ is the input voltage at the stator terminals (and control input) and $R$ is the stator winding resistance. The rotor dynamics is given by a simple integrator of the input torques, these are the load torque $\tau_{L}$ and the mechanical torque of electromechanical 
origin, which based on the assumption that the behavior of the stator windings is decoupled, is given by

$$
\tau_{e}(q, x)=\sum_{j=1}^{m} K_{j}(q) x_{j}^{2} .
$$

Thus the complete motor model is given by

$$
\begin{array}{r}
L(q) \dot{x}+K(q) \omega x+R x=u \\
J \dot{\omega}=\tau_{e}(q, x)-\tau_{L}(q, \omega)
\end{array}
$$

where $L:=\operatorname{diag}\left\{L_{j}\right\}, K:=\operatorname{diag}\left\{K_{j}\right\}, R:=\operatorname{diag}\left\{R_{j}\right\}$ and $x:=\operatorname{col}\left[x_{1}, x_{2}, x_{3}\right]^{\top}$; for further development, we remark that there exist constants $\ell_{m}, \ell_{M}$ and $k_{M}$ such that for all $q \in[-\pi, \pi]$

$$
0<\ell_{m} \leq\left|L_{j}(q)\right| \leq \ell_{M}, \quad\left|K_{j}(q)\right| \leq k_{M}
$$

Equation (2b) models the rotor dynamics; $J$ denotes the rotor inertia and $\omega$ denotes the angular velocity that is, $\omega=\dot{q}$. In spite of the simplifications made for control analysis purposes, this model is adopted in both the electrical-machines and the control research communities $-c f$. [11].

We consider a three-phase electric motor that is, $m=3$. The control goal is to design a dynamic controller for (2) whose output $u=\left[\begin{array}{lll}u_{1} & u_{2} & u_{3}\end{array}\right]^{\top}$ depending on the stator currents and rotor angular positions, such that $\omega(t)$ tracks bounded smooth reference trajectories $\omega^{*}$. We make the standing assumption that $\tau_{L}$ and $J$ are both unknown and $J \in\left[J_{m}, J_{M}\right]$ with known limits $J_{m}$ and $J_{M}$. Furthermore $\dot{\omega}^{*}$ is assumed (piecewise) constant.

\section{Control STRATEGY}

The control approach consists in applying two control loops; an outer loop to stabilize the rotor dynamics via angular position measurement and an inner loop to stabilize the stator dynamics using currents measurement. More precisely, given desired reference $\omega^{*}$ we design a desired control input $\tau_{d}$ for the mechanical equation (2b), such that $\tau_{e}^{*}=\tau_{d}$ implies that $\omega \rightarrow \omega^{*}$. To that end, we define a reference mechanical torque

$$
\tau_{e}^{*}\left(q, x^{*}\right)=\frac{1}{2}\left(K_{1}(q) x_{1}^{* 2}+K_{2}(q) x_{2}^{* 2}+K_{3}(q) x_{3}^{* 2}\right)
$$

where $x_{j}^{*}$ is a current reference trajectory for each phase and which is defined as a solution to

$$
\tau_{e}^{*}=\tau_{d}
$$

for any given $\tau_{d}$. That is, the stator control loop is to ensure that $x \rightarrow x^{*}$ so that $\tau_{e} \rightarrow \tau_{d}$ and in turn, $\omega \rightarrow \omega^{*}$. Next, we explain in detail both controllers for the rotor and the stator dynamics.

\section{A. Control of rotor velocity}

The complexity of the rotor dynamics equation resides in the fact that it is non-affine in the (virtual control) inputs $x$; such difficulty is overcome by using the torque-sharing technique -see [7], explained farther below. For the time being, let us consider that $\tau_{e}$ is a virtual control input to (2b) then, (5) implies that

$$
J \dot{\omega}=\tau_{d}-\tau_{L}+\tau_{e}-\tau_{e}^{*}
$$

For the purpose of designing the control law $\tau_{d}$ for (6) we see the latter as a simple integrator of the unknown constant $\tau_{L}$ and we consider $\tau_{e}-\tau_{e}^{*}$ as a vanishing perturbation. The choice of proportional-integral-derivative control (PID) comes naturally; furthermore, since $\omega$ is assumed to be unmeasurable, we use the $\mathrm{PI}^{2} \mathrm{D}$ controller, introduced in [9] for robot manipulators. It corresponds to a modified PID controller in which the 'derivative' term is proportional to a filtered velocity vector and double integral action, both on $e_{q}$ and $\vartheta$, is used. The fact that PID control is model-free is particularly suitable in the context of parametric uncertainty. The $\mathrm{PI}^{2} \mathrm{D}$ controller, for the rotor dynamics is defined by

$$
\begin{aligned}
\tau_{d} & =-k_{p} e_{q}-k_{d} \vartheta+\nu+\hat{J} \dot{\omega}^{*} \\
\dot{\nu} & =-k_{i}\left(e_{q}-\vartheta\right) \\
\dot{q}_{c} & =-a\left(q_{c}+b e_{q}\right) \\
\vartheta & =q_{c}+b e_{q}
\end{aligned}
$$

where $k_{p}, k_{i}, k_{d}, a, b$ are positive reals, $\hat{J} \in\left[J_{m}, J_{M}\right]$ is a constant estimate of $J$ and $e_{q}=q-q^{*}$ with

$$
q^{*}(t)=\int_{0}^{t} q^{*}(s) d s, \quad q^{*}(0)=q_{0}^{*} \in[-\pi, \pi] .
$$

Note that $\tau_{d}$ is independent on $\omega$. Since the variable to be controlled is $\omega$, the initial value of $q_{0}^{*}$ is innocuous. It is also important to remark that $\hat{J} \dot{\omega}^{*}$ is (piecewise) constant by assumption hence, its effect and that of the load torque $\tau_{L}$ may be compensated for by integral action, as a matter of fact $\nu$ converges (albeit slowly) to

$$
\nu^{*}:=\tau_{L}-\tilde{J} \dot{\omega}^{*}
$$

where $\tilde{J}=\hat{J}-J$. The last two equations in (7) correspond to the well-known and widely used 'dirty derivatives'. The nickname comes from the observation that it is equivalent to

$$
\vartheta=\frac{b}{s+a} e_{\omega}
$$

where $s$ is the Laplace variable. Note that $\vartheta$ is not a converging estimate of the velocity $e_{\omega}$ that is $\vartheta \nrightarrow e_{\omega}$, save in the limit case when the pole is placed at $-\infty$ and the DC gain $b / a=1$. Thus, using (7a) in (6), $\hat{J}=\tilde{J}+J$ and defining

$$
z:=\nu+\tilde{J} \dot{\omega}^{*}-\frac{k i}{\varepsilon} e_{q}-\tau_{L}, \quad k_{p}^{\prime}=k_{p}-\frac{k_{i}}{\varepsilon}
$$

we see that the mechanical equation becomes

$$
\left[\begin{array}{c}
\dot{e}_{q} \\
\dot{e}_{\omega} \\
\dot{\vartheta} \\
\dot{z}
\end{array}\right]=\underbrace{\left[\begin{array}{cccc}
0 & 1 & 0 & 0 \\
-k_{p}^{\prime} / J & 0 & -k_{d} / J & 1 / J \\
0 & b & -a & 0 \\
-k_{i} & -k_{i} / \varepsilon & k_{i} & 0
\end{array}\right]}_{A} \underbrace{\left[\begin{array}{c}
e_{q} \\
e_{\omega} \\
\vartheta \\
z
\end{array}\right]}_{y_{1}}+\underbrace{\left[\begin{array}{c}
0 \\
1 / J \\
0 \\
0
\end{array}\right]}_{B}
$$

where $u_{\tau}:=\tau_{e}-\tau_{e}^{*}$. Note that we can make the matrix $A$ Hurwitz by properly choosing the gains $k_{p}, k_{i}, k_{d}, a$ and $b$ without the knowledge of $J$. Consequently, the mapping $u_{\tau} \mapsto y_{1}$ is input to state stable. Indeed, the Hurwitz property 
is equivalent to the existence of $P=P^{\top}>0$ and $\gamma_{1}>|P B|$ such that $Q=-\left(A^{\top} P+P A\right)$ is positive definite and

$$
V_{1}\left(y_{1}\right)=\frac{1}{2} y_{1}^{\top} P y_{1}
$$

satisfies

$$
\begin{aligned}
\dot{V}_{1}\left(y_{1}\right) & =\frac{1}{2} y_{1}^{\top}\left(A^{\top} P+P A\right) y_{1}+y_{1}^{\top} P B u_{\tau} \\
& \leq-\frac{1}{2} y_{1}^{\top} Q y_{1}+\gamma_{1}\left|y_{1}\right|\left|\tau_{e}-\tau_{e}^{*}\right| .
\end{aligned}
$$

The control loop of the stator dynamics, explained next, is responsible for making $\left|\tau_{e}-\tau_{e}^{*}\right| \rightarrow 0$.

\section{B. Control of the stator dynamics}

We stress that Equation (9) is equivalent to (2b) provided that (5) holds for $\tau_{d}$ defined as in (7a); to ensure that $\tau_{e}^{*}\left(q, x^{*}\right)=\tau_{d}$ we need to solve the latter for $x^{*}$. To that end, we employ the so-called torque sharing technique, introduced in [7] and which leads to the definition of a reference current trajectory $x^{*}$. According to (4) we have for each $j \in\{1,2,3\}$

$$
x_{j}^{*}=\left[\frac{2 \tau_{d}}{K_{j}(q)}\right]^{1 / 2}
$$

however, for $x_{j}^{*}$ to be well-posed $K_{j}(q)$ must be different from zero and its sign must be the same as that of $\tau_{d}$. To ensure that this is the case, we exploit the physics of the reluctance machine as in [12], [7] and introduce a currentswitching policy defined by commutation piecewise constant functions $q \mapsto m_{j}$ as follows. Let the sets

$$
\begin{aligned}
& \Theta_{j}^{+}=\left\{q \in[-\pi, \pi]: s_{j}(q) \geq 0\right\} \\
& \Theta_{j}^{-}=\left\{q \in[-\pi, \pi]: s_{j}(q)<0\right\}
\end{aligned}
$$

and let $m_{j}$ be such that $\sum_{j=1}^{3} m_{j}(q)=1$,

$$
m_{j}(q)=\left\{\begin{array}{lll}
m_{j}^{+}(q) & \text { if } \quad \tau_{d} \geq 0 \\
m_{j}^{-}(q) & \text { if } \quad \tau_{d}<0 .
\end{array}\right.
$$

where

$$
\begin{array}{llll}
m_{j}^{+}(q)>0 & \forall q \in \Theta^{+}, & m_{j}^{+}(q)=0 & \forall q \in \Theta^{-}, \\
m_{j}^{-}(q)>0 & \forall q \in \Theta^{-}, & m_{j}^{-}(q)=0 & \forall q \in \Theta^{+} .
\end{array}
$$

Because the functions $s_{j}$ are sinusoids out of phase by $2 \pi / 3$, for each $q$ and $\tau_{d}$ there always exists (at least) one $j \in$ $\{1,2,3\}$ such that

$$
\frac{2 \tau_{d} m_{j}(q)}{K_{j}(q)} \in(0, \infty)
$$

Furthermore, to smoothen the phase transitions one may use smooth functions $m_{j}$ as opposed to piecewise constant and we introduce hysteresis around the switching condition $s_{j}=$ 0 that is, let

$$
x_{j}^{*}(\sigma)= \begin{cases}\sigma\left[\frac{\tau_{d} m_{j}(q)}{s_{j}(q)}\right]^{1 / 2} & \text { if }\left|s_{j}(q)\right|>\delta_{K} \\ 0 & \text { otherwise. }\end{cases}
$$

where $\delta_{K}$ is the hysteresis design parameter and

$$
\sigma=\sqrt{\frac{2}{N_{r} \ell_{1}}} .
$$

Under these conditions, $\tau_{e}^{*}\left(q, x^{*}\right)=\tau_{d}$.

The rationale to solve the tracking control problem $x_{j} \rightarrow$ $x_{j}^{*}$ builds upon the observation that under the action of the tracking control law

$$
u=L(q) \dot{x}^{*}+\omega^{*} K(q) x+R x-k_{p x} e_{x}, \quad k_{p x}>0
$$

where $e_{x}:=x-x^{*}$, the origin of the closed-loop equation

$$
L(q) \dot{e}_{x}+k_{p x} e_{x}=-K(q) x e_{\omega}, \quad e_{\omega}:=\omega-\omega^{*}
$$

is globally exponentially stable provided that $e_{\omega} \equiv 0$. Indeed, the system is reminiscent of a perturbed linear system with stable drift; to see this, note that $L(q)$ is positive definite and bounded uniformly in $q$ therefore, exponential stability of the origin of

$$
L(q) \dot{e}_{x}=-\left[R+k_{p x}\right] e_{x}
$$

is equivalent to that of the origin of

$$
\dot{e}_{x}=-\left[R+k_{p x}\right] e_{x}
$$

which holds for any positive value of the control gain $k_{p x}$. Furthermore, the system may be rendered input to state stable from the input $e_{\omega}$ provided that the gain $k_{p x}$ dominates over the input "gain" function $K(q) x$; note that this is feasible as $q$ and $x$ are measured states.

Although simple and appealing, the control law (14) is not implementable since $\dot{x}^{*}$ depends on the unmeasured velocity. Indeed,

$$
\dot{x}_{j}^{*}= \begin{cases}\sigma \alpha_{j} \rho_{j}+\sigma \alpha_{j} \delta_{j} e_{\omega} & \text { if }\left|s_{j}(q)\right|>\delta_{K} \\ 0 & \text { otherwise. }\end{cases}
$$

where $\alpha=\operatorname{diag}\left\{\alpha_{1}, \alpha_{2}, \alpha_{3}\right\}$ is a diagonal matrix, $\rho=$ $\left[\begin{array}{lll}\rho_{1} & \rho_{2} & \rho_{3}\end{array}\right]^{\top}$ and $\delta=\left[\begin{array}{lll}\delta_{1} & \delta_{2} & \delta_{3}\end{array}\right]^{\top}$ are defined as

$$
\begin{aligned}
\alpha_{j} & =\frac{1}{2}\left[\frac{m_{j} \tau_{d}}{s_{j}}\right]^{-1 / 2} \\
\rho_{j} & =\frac{1}{s_{j}}\left[\left(k_{d} a+k_{i}\right) m_{j} \vartheta-k_{i} m_{j} e_{q}+\frac{\partial m_{j}}{\partial q} \tau_{d} \omega^{*}\right]-\frac{N_{r} c_{j}}{s_{j}^{2}} \tau_{d} \omega^{*} \\
\delta_{j} & =\frac{1}{s_{j}}\left[-m_{j}\left(k_{p}+k_{d} b\right)+\frac{\partial m_{j}}{\partial q} \tau_{d}\right]-\frac{N_{r} c_{j}}{s_{j}^{2}} \tau_{d} .
\end{aligned}
$$

Notice that $\alpha, \rho$ and $\delta$ are functions of known parameters and measurable variables. Since $e_{\omega}$ is not measurable, we introduce the following control law which is reminiscent of $u$ defined in (14) except that we drop the term $\alpha \delta_{j} e_{\omega}$ in the definition of $\dot{x}^{*}$ that is,

$$
u=L(q) \sigma \alpha \rho+\omega^{*} K(q) x+R x^{*}-k_{p x} e_{x},
$$

where $e_{x}:=x-x^{*}$. Note that, in view of (16), (17) is equivalent to

$$
u=L(q) \dot{x}^{*}+\omega^{*} K(q) x+R x^{*}-k_{p x} e_{x}-\sigma L(q) \alpha \delta e_{\omega}
$$

then, the closed-loop equation (2a) with (18) yields

$$
L(q) \dot{e}_{x}=-\left[R+k_{p x}\right] e_{x}-[K(q) x+\sigma L(q) \alpha \delta] e_{\omega}
$$

which is also reminiscent of a perturbed linear system with stable drift; in this case, the input gain

$$
g(t, x, \xi):=[K(q(t)) x+\sigma L(q(t)) \alpha(t, \xi) \delta(t, \xi)]
$$


depends on known quantities and measured the states $\xi:=$ $\left[e_{q} \vartheta z\right]^{\top}$ and since $L, K, m_{j}, \frac{\partial m_{j}}{\partial q}$ and $\omega^{*}$ are uniformly bounded, there exists a non-decreasing function $\gamma_{2}: \mathbb{R}_{\geq 0} \times$ $\mathbb{R}_{\geq 0} \rightarrow \mathbb{R}_{\geq 0}$ such that

$$
|g(t, x, \xi)| \leq \gamma_{2}(|\xi|,|x|) \text {. }
$$

Therefore, it may be established that (19) is input-to-state stable with respect to the input $e_{\omega}$, for an appropriate choice of the gain $k_{p x}$ dependent on $\gamma_{2}$, hence on $|\xi|$ and $|x|$.

\section{MAIN RESUlTS}

The developments in the previous section indicate that the closed-loop system (9), (19) consists in the interconnection of two input-to-state stable systems for which the feedback gains may be adjusted to ensure global exponential stability. The proof of this claim constitutes our first contribution. Then, we show that in the case that all parameters are unknown, a certainty-equivalence adaptive controller ensures the convergence of the tracking errors to zero.

\section{A. Robust control and system parameterization}

Let $C(q):=\operatorname{diag}\left\{c_{j}(q)\right\}, S(q):=\operatorname{diag}\left\{s_{j}(q)\right\}$ then, $L(q)=\ell_{0} I-\ell_{1} C(q)$ and $K(q)=\ell_{1} N_{r} S(q)$. With this notation, the control law (17) may be written as

$$
u=\ell_{0} \sigma \alpha \rho-\ell_{1} C(q) \sigma \alpha \rho+\omega^{*} \ell_{1} N_{r} S(q) x+R x^{*}-k_{p x} e_{x}
$$

which is linear in the physical parameters $\ell_{0}, \ell_{1}$ and $R$ therefore,

$$
\begin{aligned}
& u=\Psi(t, \xi)^{\top} \Theta-k_{p x} e_{x},
\end{aligned}
$$

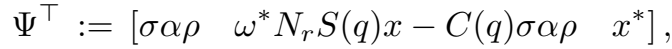

$$
\begin{aligned}
& \Theta:=\left[\begin{array}{lll}
\ell_{0} & \ell_{1} & R
\end{array}\right]^{\top} .
\end{aligned}
$$

We stress that $\Psi$ is a function of $t$ and the measured closedloop states $\xi$; indeed, one should read $q(t)$ and $x(t)$ in place of $q$ and $x$ while $\alpha, \rho$ and $x^{*}$ are functions of $t$ and $\xi$.

Proposition 1 Consider the system (2) in closed loop with the controller (20), -see also (12) and (7). Let $a, b, k_{p}, k_{d}$, $k_{i}$ and $\varepsilon>k_{i}$ be positive real numbers such that $A$ in (9) is Hurwitz. Then, there exist a real number $k_{p x}^{\prime}>0$ and a nondecreasing function $k_{p x}^{\prime \prime}: \mathbb{R}_{\geq 0}^{3} \rightarrow \mathbb{R}_{\geq 0}$ such that defining

$$
k_{p x}=k_{p x}^{\prime}+k_{p x}^{\prime \prime}\left(|\xi|,|x|,\left|x^{*}\right|\right),
$$

the origin of the closed-loop system $\left\{\left[y_{1}, e_{x}\right]=[0,0]\right\}$ is uniformly globally exponentially stable.

Proof: The rotor dynamical equation is equivalent to (6) for any $\tau_{e}^{*}$, for the purpose of proof let $\tau_{e}^{*}=\tau_{e}^{*}\left(q, x^{*}\right)$ that is,

$$
\tau_{e}^{*}:=\frac{1}{2} \sum_{j=1}^{3} K_{j}(q)\left|\hat{x}_{j}^{*}\right|^{2}
$$

so (5) holds in view of the definition of $x^{*}$, this implies in turn that (6) is equivalent to (9). Now, a direct computation shows that,

$$
\left|\tau_{e}-\tau_{e}^{*}\right| \leq \frac{k_{M}}{2}\left|e_{x}^{\top}\left(x+x^{*}\right)\right|
$$

On the other hand, substituting $u$ from (20) in (2a) we obtain the closed-loop equation

$$
L(q) \dot{e}_{x}=-\left[R+k_{p x}\right] e_{x}-g(t, x, \xi) e_{\omega} .
$$

Next, let $Q=Q_{1}+Q_{2}$ where $Q_{1}$ is positive definite, $Q_{2}=Q_{2}^{\top} \geq 0$ and there exists $q_{m}>0$ such that $q_{m}\left|y_{1}\right|^{2} \leq$ $\frac{1}{2} y_{1}^{\top} Q_{1} y_{1}$ and $y_{1}^{\top} Q_{2} y_{1} \geq \varepsilon_{1}(b-1) e_{\omega}^{2}$. Let the Hurwitz assumption on $A$ generate for such matrix $Q$, a positive definite matrix $P$ and reals $p_{M} \geq p_{m}>0$ such that $V_{1}$ in (10) satisfies

$$
p_{m}\left|y_{1}\right|^{2} \leq V_{1}\left(y_{1}\right) \leq p_{M}\left|y_{1}\right|^{2}
$$

and the derivative of $V_{1}$ satisfies (11). Then, from (11) and (22) we obtain

$\dot{V}_{1}\left(y_{1}\right) \leq-q_{m}\left|y_{1}\right|^{2}+\frac{k_{M} \gamma_{1}}{2}\left|y_{1}\right|\left|e_{x}^{\top}\left(x+x^{*}\right)\right|-\varepsilon_{1}(b-1) e_{\omega}^{2}$.

Next, consider the function $V_{2}: \mathbb{R}_{\geq 0} \times \mathbb{R}^{3} \rightarrow \mathbb{R}_{\geq 0}$ defined by

$$
V_{2}\left(t, e_{x}\right)=\frac{1}{2} e_{x}^{\top} L(q(t)) e_{x}
$$

which is positive definite and radially unbounded since $|L(q)|$ is uniformly bounded and positive definite actually, in view of (3),

$$
\frac{\ell_{m}}{2}\left|e_{x}\right|^{2} \leq V_{2}\left(t, e_{x}\right) \leq \ell_{M}\left|e_{x}\right|^{2}
$$

The total derivative of $V_{2}$ along the trajectories of (23) satisfies

$$
\begin{aligned}
\dot{V}_{2}\left(t, e_{x}\right) \leq-e_{x}^{\top} & {\left[\left(k_{p x}-\gamma_{2}^{2} \lambda_{1}-\frac{k_{M}}{2} \omega^{*}\right) I\right.} \\
& \left.-\frac{\lambda_{3}}{2} e_{x}^{\top} K(q) e_{x} K(q)\right] e_{x}+\left(\frac{1}{\lambda_{1}}+\frac{1}{\lambda_{3}}\right) e_{\omega}^{2}
\end{aligned}
$$

for which we used $\overparen{L(q)}=K(q)\left[e_{\omega}+\omega^{*}\right], e_{x}^{\top} K(q) e_{x} e_{\omega} \leq$ $\left(1 / \lambda_{3}\right) e_{\omega}^{2}+\lambda_{3}\left[e_{x}^{\top} K(q) e_{x}\right]^{2}$ and $e_{x}^{\top} g e_{\omega} \leq \lambda_{1} \gamma_{2}^{2}\left|e_{x}\right|^{2}+$ $\left(1 / \lambda_{1}\right) e_{\omega}^{2}$. Thus, the total derivative of the Lyapunov function $V_{3}:=V_{1}+V_{2}$ along the closed-loop trajectories satisfies

$$
\begin{aligned}
\dot{V}_{3} \leq- & {\left[\varepsilon_{1}(b-1)-\left(\frac{1}{\lambda_{1}}+\frac{1}{\lambda_{3}}\right)\right] e_{\omega}^{2}-\left[q_{m}-\frac{\lambda_{2} k_{M} \gamma_{1}}{2}\right]\left|y_{1}\right|^{2} } \\
- & {\left[k_{p x}-\lambda_{1} \gamma_{2}^{2}-\frac{k_{M}}{2}\left(\omega^{*}+\lambda_{3} k_{M}\left|e_{x}\right|^{2}\right.\right.} \\
& \left.\left.+\frac{\gamma_{1}}{\lambda_{2}}\left|x+x^{*}\right|^{2}\right)\right]\left|e_{x}\right|^{2}
\end{aligned}
$$

We see that for any given $q_{m}, \varepsilon_{1}$ and $b$ there exist positive numbers $\lambda_{1}, \lambda_{2}, \lambda_{3}$ and $c$ such that defining $k_{p x}^{\prime}>0$ and $^{2}$

$$
\begin{gathered}
k_{p x}^{\prime \prime}:=\frac{k_{M}}{2}\left(\omega^{*}+\lambda_{3} k_{M}\left|e_{x}\right|^{2}+\frac{\gamma_{1}}{\lambda_{2}}\left|x+x^{*}\right|^{2}\right) \\
+\lambda_{1} \gamma_{2}(|\xi|,|x|)^{2}
\end{gathered}
$$

we have

$$
\dot{V}_{3}\left(t, y_{1}, e_{x}\right) \leq-c\left[\left|y_{1}\right|^{2}+\left|e_{x}\right|^{2}\right] .
$$

The result follows.

${ }^{2}$ Notice that $\left|x^{*}\right|^{2}=\mathcal{O}\left(\tau_{d}\right)$ hence, it is bounded by a non-decreasing function of order $\mathcal{O}(|\xi|)$. 


\section{B. Control under full parametric uncertainty}

In the previous section we assumed that the rotor inertia $J$ and the load-torque are unknown and we established uniform global exponential stability of the origin. The latter includes $\left\{\left[y_{1} e_{x}\right] \in \mathbb{R}^{7}: e_{\omega}=0, e_{x}=0\right\}$. This implies that currents follow exponentially any reference $x^{*}$ as defined in (12) and the rotor angular velocity is stabilized at the desired reference $\omega^{*}$. Note that this holds regardless of the value of $\sigma$. Now we relax the assumption that $L$ and $R$ are known that is, we assume that the physical parameters $R, \ell_{0}, \ell_{1}$ and hence $\sigma\left(\ell_{1}\right)$, are also unknown.

Let us redefine the reference for the stator currents by using the constant

$$
\hat{\sigma}=\sqrt{\frac{2}{N_{r} \hat{\ell}_{1}(0)}}
$$

where $\hat{\ell}_{1}(0)$ denotes the best estimate available of $\ell_{1}$. That is, we use the reference $\hat{x}^{*}:=x^{*}(\hat{\sigma})$ where $x^{*}(\cdot)$ is defined in (12) and to avoid an over-parameterization, we choose to estimate online only $\Theta:=\left[\ell_{0}, \ell_{1}, R\right]$.

Proposition 2 Consider the system (2) in closed loop with the certainty-equivalence controller

$$
u=\Psi\left(t, \xi, \hat{e}_{x}\right)^{\top} \hat{\Theta}-k_{p x} \hat{e}_{x}, \quad \hat{e}_{x}:=x-\hat{x}^{*}
$$

and the adaptation law

$$
\dot{\hat{\Theta}}=-k_{\theta} \Psi\left(t, \xi, \hat{e}_{x}\right) \hat{e}_{x}, \quad k_{\theta}>0
$$

under the conditions of Proposition 1 with $\hat{x}^{*}$ in place of $x^{*}$ in (21). Define $\tilde{\Theta}:=\hat{\Theta}-\Theta$. Then, the origin of the closedloop system, $\left\{\left[y_{1}, \hat{e}_{x}, \tilde{\Theta}\right]=[0,0,0]\right\}$ is uniformly globally stable and the tracking errors $y_{1}$ and $\hat{e}_{x}$ satisfy

$$
\lim _{t \rightarrow \infty}\left|y_{1}(t)\right|=0 \quad \lim _{t \rightarrow \infty}\left|\hat{e}_{x}(t)\right|=0 .
$$

Proof: The closed-loop system is given by equations (9), (23) with $\hat{e}_{x}$ in place of $e_{x}$. Consider the Lyapunov function $V: \mathbb{R}_{\geq 0} \times \mathbb{R}^{4} \times \mathbb{R}^{3} \times \mathbb{R}^{3} \rightarrow \mathbb{R}_{\geq 0}$ defined by

$$
V\left(t, y_{1}, \hat{e}_{x}, \tilde{\Theta}\right):=V_{3}\left(t, y_{1}, \hat{e}_{x}\right)+\frac{1}{2 k_{\theta}}|\tilde{\Theta}|^{2}
$$

which is positive definite and radially unbounded under the conditions of Proposition 1. Following the steps of proof of Proposition 1, using

$$
u=\Psi\left(t, \xi, \hat{e}_{x}\right)^{\top} \Theta-k_{p x} \hat{e}_{x}+\Psi\left(t, \xi, \hat{e}_{x}\right)^{\top} \tilde{\Theta},
$$

and replacing $x^{*}$ in (24) and (25) with $\hat{x}^{*}$, yields

$$
\dot{V}\left(t, y_{1}, \hat{e}_{x}, \tilde{\Theta}\right) \leq-c\left[\left|y_{1}\right|^{2}+\left|\hat{e}_{x}\right|^{2}\right] \leq 0
$$

in place of (26). Uniform global stability of the origin follows by integrating $\dot{V} \leq 0$ along the closed-loop trajectories. In addition, by integrating the first inequality in (29) we see that $y_{1} \in \mathcal{L}_{2}, \hat{e}_{x} \in \mathcal{L}_{2}$. Furthermore, uniform global stability implies uniform global boundedness hence $y_{1} \in \mathcal{L}_{\infty}, \hat{e}_{x} \in$ $\mathcal{L}_{\infty}$; in view of (3), a simple inspection at the closed-loop equations shows that $\dot{y}_{1} \in \mathcal{L}_{\infty}$ and $\dot{\hat{e}}_{x} \in \mathcal{L}_{\infty}$. The result follows, invoking Barbalăt's lemma.

\section{Simulation Results}

With aim at evaluating the controller of Proposition 2 we have performed some numerical simulations in SIMULINK ${ }^{\mathrm{TM}}$ of MATLAB ${ }^{\mathrm{TM}}$, using the parameters presented in [10] $R=$ $0.3[\Omega], \ell_{0}=24[\mathrm{mH}], \ell_{1}=19[\mathrm{mH}]$ and $N r=25$. The control gains are $a=1500, b=3200, k_{p}=1050, k_{i}=$ $5 \times 10^{-4}, k_{d}=1000, k_{p x}^{\prime}=150, k_{p x}^{\prime \prime}=0$ and $k_{\theta 1}=15$. We stress that for implementation purpose, we use a constant value of $k_{p x}$ even though the sufficient condition for global stability is that this gain depends on the measured states. The reason to fix $k_{p x}^{\prime \prime}=0$ is to avoid high values in the input voltages which bring the converters into saturation.

The experiment consist in imposing a smoothed step speed reference defined by

$$
\omega^{*}(t)=\omega_{0}^{*}+\frac{\left(\omega_{f}^{*}-\omega_{0}^{*}\right)}{2}(1+\tanh (t-T))
$$

with the final desired velocity set on $190[\mathrm{rad} / \mathrm{s}]$. The load torque is piecewise constant, for the first half of the simulation time $\tau_{L}=0.1$ then, it is increased by $50 \%$ at $t=3.5 \mathrm{~s}$.

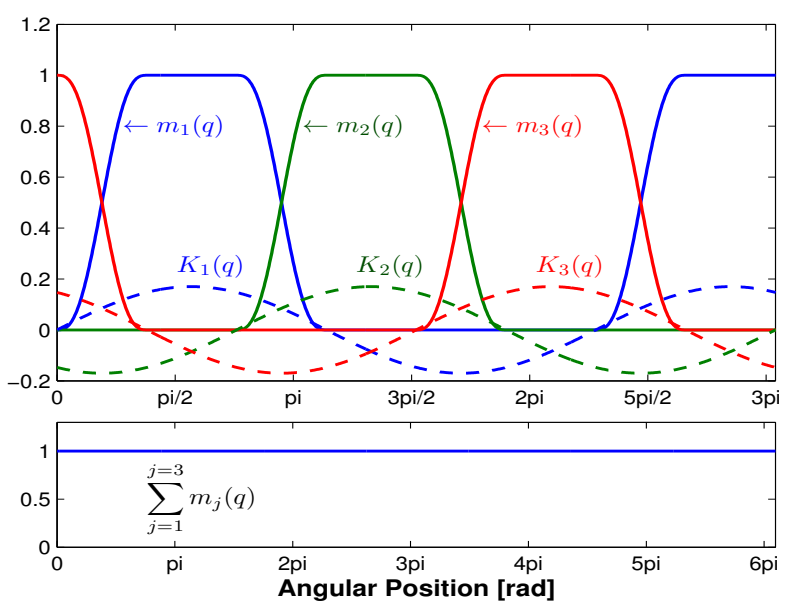

Fig. 1. Graph of the commutation functions $q \mapsto m_{j}$

The commutation functions $m_{j}:[0,2 \pi) \rightarrow \mathbb{R}_{\geq 0}$ are illustrated in Fig. 1 and are defined as follows. Let

$$
f(x)=10 \frac{x^{3}}{\theta_{m}^{3}}-15 \frac{x^{4}}{\theta_{m}^{4}}+6 \frac{x^{5}}{\theta_{m}^{5}}
$$

then, for $j=1,2,3$ and $q_{1}=\bmod \left(q_{0}, \pi / 4\right), q_{2}=\bmod \left(q_{0}-\right.$ $\pi / 12, \pi / 4), q_{3}=\bmod \left(q_{0}+\pi / 12, \pi / 4\right)$,

$$
\begin{gathered}
m_{j}^{+}(\theta)= \begin{cases}f_{j}\left(q_{a j}\right) & 0<q_{a j} \leq \frac{\pi}{3 N_{r}} \\
1 & \frac{\pi}{3 N_{r}}<q_{a j} \leq \frac{2 \pi}{3 N_{r}} \\
1-f_{j}\left(q_{a j}\right) & \frac{2 \pi}{3 N_{r}}<q_{a j} \leq \frac{\pi}{N_{r}} \\
0 & \text { otherwise }\end{cases} \\
m_{j}^{-}(\theta)= \begin{cases}f_{j}\left(q_{a j}\right) & \frac{\pi}{N_{r}}<q_{a j} \leq \frac{4 \pi}{3 N_{r}} \\
1 & \frac{4 \pi}{3 N_{r}}<q_{a j} \leq \frac{5 \pi}{3 N_{r}} \\
1-f_{j}\left(q_{a j}\right) & \frac{5 \pi}{3 N_{r}}<q_{a j} \leq \frac{2 \pi}{N_{r}} \\
0 & \text { otherwise }\end{cases}
\end{gathered}
$$


The operator $\bmod$ resets $q$ i.e., $q_{j}=\bmod \left(\beta_{1}, \beta_{2}\right)$ is such that $q_{j}(0)=\beta_{1}$ and $q_{j}$ is reset to the latter when $q_{j}(t)=\beta_{2}$.

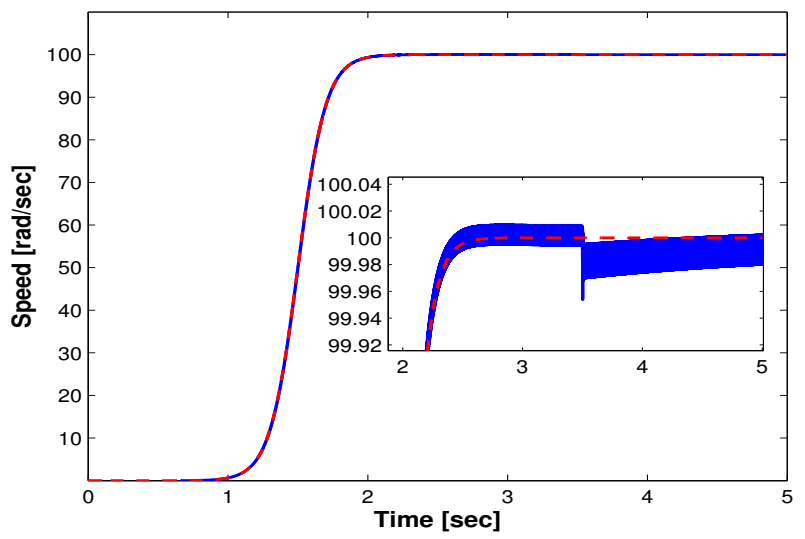

Fig. 2. Velocity Tracking

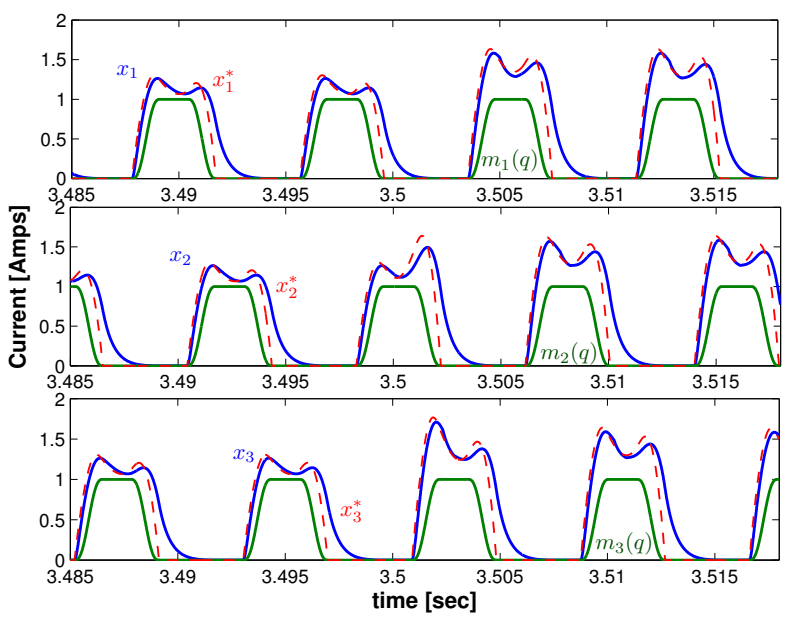

Fig. 3. Electric current around $t=3.5 \mathrm{~s}$ when the load-torque increases. The actual currents $x_{j}$ as well as the reference trajectories $\hat{x}^{*}$ and the commutation signals $m_{j}(q(t))$ are showed to be in synchrony

In Fig. 2 is depicted the system's response; note the perfect tracking of the angular velocity and the good performance. In Fig. 3 we show a zoomed window on the three stator currents and their references. The commutations due to the sharing torque approach may be clearly appreciated, as well as the effect of the load torque increase at $t=3.5 \mathrm{~s}$, the motor requires more electrical current in order to remain at the required velocity set-point. The corresponding input voltages are depicted in Fig. 4.

\section{CONCLUDING REMARKS}

We presented an adaptive controller for the switchedreluctance motor, considering both the stator and rotor dynamics. Our control approach exploits the physical properties of the machine, relying on torque-sharing approach. Ongoing research focuses on the sensorless control problem that is, avoiding the use of position measurements.

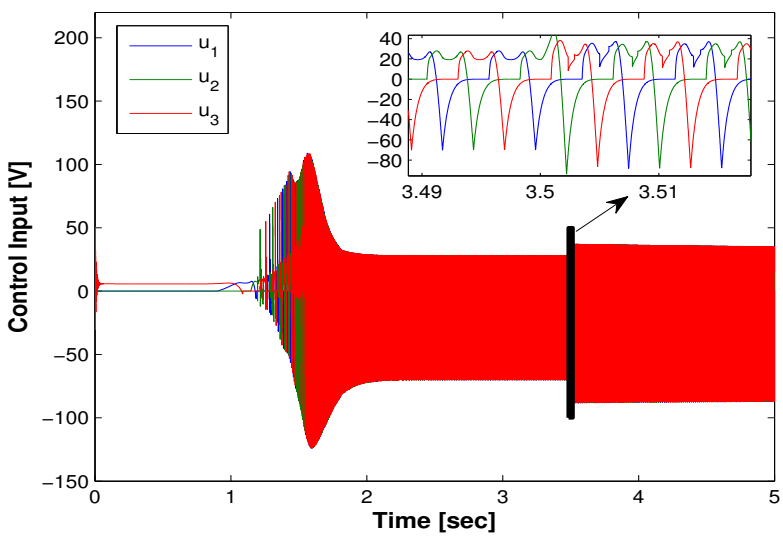

Fig. 4. Voltage control inputs $u_{j}$

\section{Acknowledgments}

The work of G. Espinosa is supported by DGAPA-UNAM under grant IN11121. E. Chumacero benefits from a scholarship by CONACyT, Mexico.

\section{REFERENCES}

[1] G. LI, J. Ojeda, E. Hoang, M. Gabsi, and M. Lécrivain. A new method of current density distribution for switching reluctance machine to increase average output torque. In PCIM, Shanghai, China, 2009.

[2] Yi Zheng, Hexu Sun, Yan Dong, and Zhaoming Lei. The research of stator current oriented method of switched reluctance motor. In Control and Decision Conference, 2008. CCDC 2008. Chinese, pages $3184-3188$, july 2008.

[3] Wang Xiaoyuan, Pan Hao, and Guo Zhi. Double switch reluctance motors synchronization control system. In Control and Decision Conference, 2008. CCDC 2008. Chinese, pages 3754-3756, july 2008.

[4] H. Peyrl, G. Papafotiou, and M. Morari. Model predictive torque control of a switched reluctance motor. In Industrial Technology, 2009. ICIT 2009. IEEE International Conference on, pages 1 -6, feb. 2009.

[5] L.L.N. dos Reis, F. Sobreira, A.R.R. Coelho, O.M. Almeida, J.C.T. Campos, and S. Daher. Identification and adaptive speed control for switched reluctance motor using DSP. In Power Electronics Conference, 2009. COBEP '09. Brazilian, pages 836 -841, 27 2009. oct. 12009.

[6] G. Espinosa-Perez, P. Maya-Ortiz, M. Velasco-Villa, and H. SiraRamirez. Passivity-based control of switched reluctance motors with nonlinear magnetic circuits. Control Systems Technology, IEEE Transactions on, 12(3):439 - 448, may 2004.

[7] D. Taylor. Pulse-width modulated control of electromechanical systems. IEEE Trans. Automat. Contr., AC-37:524-528, 1992.

[8] R. Milman and S.A. Bortoff. Observer-based adaptive control of a variable reluctance motor: Experimental results. Control Systems Technology, IEEE Transactions on, 7(5):613 -621, sep 1999.

[9] R. Ortega, A. Loría and R. Kelly. A semiglobally stable output feedback PI2D regulator for robot manipulators. IEEE Trans. on Automat. Contr., 40(8):1432-1436, 1995.

[10] M. Ilic"-Spong, R. Marino, S.M. Peresada, and D. Taylor. Feedback linearizing control of switched reluctance motors. Automatic Control, IEEE Transactions on, 32(5):371-379, May.

[11] R. Krishnan. Switched Reluctance Motor Drives. CRC Press, 2001.

[12] M. Ilic-Spong, R. Marino, S. Peresada, and D. Taylor. Feedback linearizing control of switched reluctance motors. IEEE Trans. Automat. Contr., AC-32:371-379, 1987. 